\title{
WORKSHOP REPORT: ORGAN PRESERVATION STRATEGIES IN ADVANCED HEAD AND NECK CANCER-CURRENT STATUS AND FUTURE DIRECTIONS
}

\author{
Gregory T. Wolf, MD, ${ }^{1}$ Arlene Forastiere, $M D,{ }^{2}$ Kian Ang, MD, ${ }^{3}$ Bruce Brockstein, MD, ${ }^{4}$ \\ Barbara Conley, MD, ${ }^{5}$ Helmuth Goepfert, MD, ${ }^{3}$ Dennis Kraus, MD, ${ }^{6}$ \\ Jean-Louis Lefebvre, MD, ${ }^{7}$ Thomas F. Pajak, PhD, ${ }^{8}$ David Pfister, MD, ${ }^{6}$ Susan Urba, MD ${ }^{1}$ \\ ${ }^{1}$ Department of Otolaryngology, University of Michigan, 1904TC/1500 E. Medical Center Drive, \\ Ann Arbor, Michigan 48109 \\ ${ }^{2}$ The Johns Hopkins Oncology Center, Medical Oncology, Baltimore, Maryland \\ ${ }^{3}$ M.D. Anderson Cancer Center, Houston, Texas \\ ${ }^{4}$ University of Chicago Medical Center, Chicago, Illinois \\ ${ }^{5}$ National Institute of Health, National Cancer Institute, Rockville, Maryland \\ ${ }^{6}$ Memorial Sloan Kettering Cancer Center, New York, New York \\ ${ }^{7}$ Centre Oscar Lambret, Lille, France \\ ${ }^{8}$ American College of Radiology, Philadelphia, Pennsylvania
}

\section{Accepted 11 May 1999}

Keywords: organ preservation; larynx/cancer; chemotherapy; radiation

Since the first Research Conference on Head and Neck Cancer (Rosslyn, Virginia 1980), significant and systematic progress has been evident in the quality and quantity of fundamental laboratory and clinical research exploring the role of chemotherapy in the management of head and neck squamous cell carcinoma. The results of numer-

\section{Correspondence to: G. T. Wolf}

Presented at the Fifth Research Workshop on the Biology, Prevention and Treatment of Head and Neck Cancer, McLean, Virginia, August 27-30, 1998. Head \& Neck 21: 689-693, 1999

CCC 1043-3074/99/080689-05

(C) 1999 John Wiley \& Sons, Inc. ous randomized trials incorporating chemotherapy with conventional surgery and radiation have been reported consisting of more than 10,000 treated patients. The genetic basis of cancer growth, resistance to chemotherapy or radiation, and the metastatic behavior of cancers are beginning to be defined. Advances in head and neck surgical reconstruction and radiation techniques have been developed that offer promise of improved local control and reduced functional debility. Despite these advances, overall survival rates are largely unchanged. Head and neck oncology remains at a critical crossroads in defining a role for chemotherapy in the routine management of patients with advanced, potentially curable cancers. For such patients, the best treat- 
ment typically requires functionally or cosmetically debilitating surgery to achieve less than $50 \%$ 5-year cure rates.

The challenge of trying to develop treatment paradigms that address functional and quality of life (QOL) issues by incorporating chemotherapy into multimodal treatment strategies has been addressed initially by organ preservation approaches pioneered for advanced larygeal cancer and recently extended to patients with hypopharygeal and tongue base cancers, who would typically undergo a total laryngectomy. At the Fifth Research Workshop on the Biology, Prevention and Treatment of Head and Neck Cancer (McLean, Virginia, August 27-30, 1998), a special workshop was convened to discuss the current status of such clinical research and try to reach consensus on a number of practical and theoretical issues facing the use of organ preservation strategies, the design of future trials, and the goals of organ preservation (Table 1).

Barbara Conley, MD, National Cancer Institute, Bethesda, Maryland) opened the workshop by presenting a summary of the ongoing NCIsupported trials of organ preservation. Arlene Forastiere, MD, (Johns Hopkins University, Baltimore, Maryland) presented an update of RTOG 91-11, which is an intergroup three-arm clinical trial comparing sequential induction chemotherapy followed by radiation with concurrent chemoradiation or radiation alone as an organpreservation approach for stage III and limited stage IV laryngeal cancers. Expected accrual for this study is more than 500 patients and should be completed in late 1999. This trial should demonstrate how much benefit is derived by adding chemotherapy to radiation and whether an induction or concomitant approach for combining chemotherapy with radiation is better. Jean-Louis Lefebvre, MD (Centre Oscar Lambret, Lille, France) presented updated survival data from the EORTC study of organ preservation in patients with cancers of the hypopharynx and epilarynx. With 6-year follow-up, survival rates are the

Table 1. Goals of organ preservation is advanced laryngeal cancer

Cure the cancer

Minimize functional/physical deficits

Minimize length and morbidity of intensive treatment

Optimize detection and prevention of recurrent and/or secondary disease

Early, rapid rehabilitation same for patients treated with primary laryngopharyngectomy or patients randomly assigned to induction chemotherapy ( $2-3$ cycles) following which clinical complete responders receive definitive radiation and nonresponders undergo surgery. Patterns of failure posttreatment were also similar between the randomized treatment groups. Gregory T. Wolf, MD (University of Michigan, Ann Arbor, Michigan) presented an update of 10-year survival data from the VA Laryngeal Cancer Study CSP \#268, which was the first prospective randomized clinical trial of organ preservation. Long-term results confirm similar 10 -year survival rates of $25 \%$ (chemotherapy) vs $30 \%$ (surgery) for patients randomized to induction chemotherapy and radiation (for responders) vs initial laryngectomy and postoperative radiation, respectively. The power of this randomized trial for detecting a 15\% difference in survival between arms was $90 \%$ at 8 years and $87 \%$ at 10 years. The long-term larynx preservation rates remain stable with two-thirds of the survivors having an intact larynx. At 10 years, QOL analysis reveals better QOL scores for the surviving patients in the groups randomized to induction chemotherapy in every quality of health domain except speech, which was significantly poorer for all patients compared with nonlaryngeal head and neck cancer patients and similar between the two treatment groups. This contrasts with objective measures of speech production, which were significantly better in the chemoradiation group at 2 years posttreatment. No differences in overall survival were evident comparing salvage surgery patients to patients with successful organ preservation.

During the workshop, conference participants and panelists were challenged with trying to answer two questions: is organ preservation with chemotherapy and radiation an accepted alternative for laryngectomy for patients with stage III or IV laryngeal cancer; and should clinical trials of organ preservation with chemotherapy and radiation be undertaken for nonlaryngeal sites? In addressing these questions, a number of issues were raised that could influence the design of future trials (Table 2).

The first half of the workshop focused on practical aspects of organ preservation treatment approaches in advanced laryngeal cancer, including patient selection, management of the neck, assessment of tumor response, and issues relevant to surgical salvage. Panelists uniformly agreed that any patient for whom total laryngectomy was 


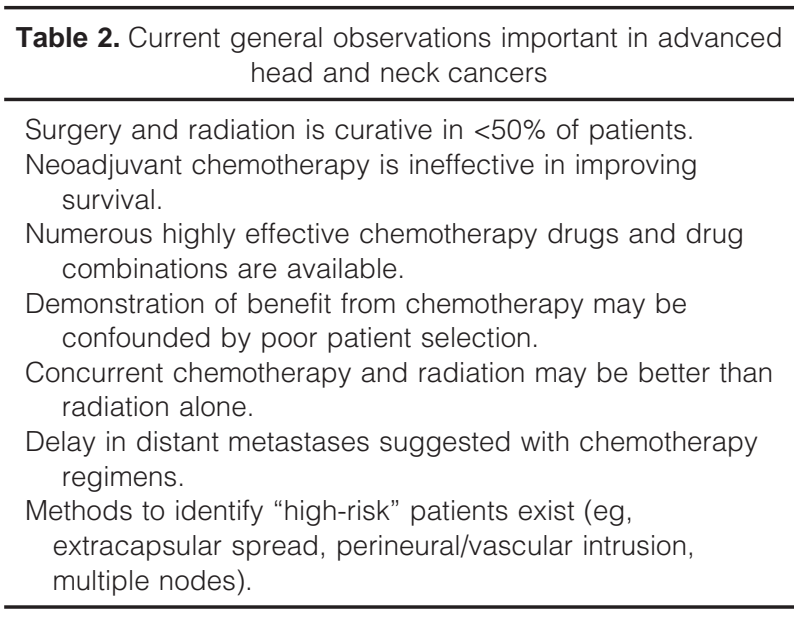

being considered should be a candidate for an organ preservation approach. Helmuth Goepfert, MD (M.D. Anderson, Houston, Texas) believed that advanced T4 tumors with extensive destruction of thyroid or cricoid cartilage should be excluded, whereas patients with bulky neck disease should not. Dennis Kraus, MD, (MSKCC, New York, New York) agreed and added that significant family support and patient reliability for comprehensive follow-up are critically important. General concern was expressed that coordination of care and interdisciplinary communication were vital to the success of combined chemoradiation regimens, particularly when surgical salvage could be such an important part of the success of the treatment. Kian Ang, MD (M.D. Anderson, Houston, Texas) expressed concerns that control of the quality of radiation and limiting treatment breaks was important. Jean Louis Lefebvre, MD (Centre Oscar Lambret, Lille, France) indicated that some patients previously considered for total laryngectomy may have tumors amenable to supracricoid partial laryngectomy, but that these procedures require special surgical expertise and careful patient selection. He did not believe that such extended partial laryngectomy procedures were wise as salvage procedures after chemoradiation. Prior tracheostomy was not believed to be a contraindication to attempted organ preservation, although it was associated with a lower success rate. Limited data that suggested that success rates may be better for chemoradiation approaches for supraglottic primary than glottic cancers were discussed.

Who among the treatment team should present the therapeutic options to the patient? Consensus was expressed that the surgeon should present the options and that patients must be in- formed of various therapeutic alternatives. These options now often include some variations of subtotal laryngectomy, radiation therapy alone, endoscopic laser resection, combined chemotherapy and radiation, and total laryngectomy. These patients should also be referred to a radiation and a medical oncologist for further discussion of options. It was strongly expressed that the surgeon has the primary responsibility for pretreatment evaluation, serial tumor assessments, and posttreatment lifelong follow-up.

The assessment of tumor extent before and after treatment was discussed. Both radiologic imaging (CT/MRI) and clinical examination were believed to be important and should be included with all patients, although some panelists favored clinical examination over radiologic examination. The use of office stroboscopic examinations and photodocumentation was viewed as useful. Helmuth Goepfert, MD (M.D. Anderson, Houston, Texas) indicated that response assessment after induction chemotherapy may not be important and that perhaps all patients should go on to complete the chemo/radiation protocol and reserve salvage surgery for failures of radiation. Lefebvre indicated that it is sometimes difficult to define a $50 \%$ (partial) response to determine who should go on to radiation. Most studies, however, have indicated that a CR (complete) response to induction chemotherapy is the strongest predictor of success. Some investigators now require a CR rather than a PR for patients to undergo definitive radiation. Work is also underway to evaluate changes in 3-dimensional tumor volumes after chemotherapy, which may provide a better indication of response and prognosis than changes in surface dimensions.

Appropriate management of the neck was reiterated as one of the most important treatment objectives in advanced laryngeal cancer, particularly for patients with supraglottic cancers. Dr. Goepfert (M.D. Anderson, Houston, Texas) generally recommends completing chemotherapy and radiation when clinical positive nodes are present and only perform neck dissection if residual palpable disease is present. Dennis Kraus (MSKCC, New York, New York) believed that most N1 disease that was a CR after chemotherapy could be observed after radiation therapy, but this could be worrisome if the initial nodes were N2 or N3. He recommends neck dissection for any palpable residual neck nodes 6 weeks after radiation. At the University of Michigan, neck dissections have been done during the interval between induction 
chemotherapy and radiation therapy for residual disease $(<\mathrm{CR}$ after chemotherapy), and this approach has proven effective and safe. The new EORTC trials allow neck dissection before or after radiation. Dr Lefebvre believes that necks that may be difficult to evaluate in follow-up might better be dissected before radiation. The consensus of the workshop participants was that neck management needed to be individualized and that best timing of neck dissection was yet to be decided. Routine dissections for bulky neck disease were favored by some panelists; however, most agreed that planned dissection for palpable residual neck nodes is important.

Finally, the group tackled the question of whether new organ preservation trials for nonlaryngeal sites should be conducted. Many and varied opinions were presented with enthusiasm for specific sites, including oropharynx (Lefebvre, Kraus), paranasal sinus, oral tongue (Ang), and tongue base (Urba). The medical oncologists (Forastiere, Conley, Urba) believed such trials were justified and should be performed but would be complex to design and that it might be difficult to get the support of surgeons in such trials.

The second half of the Organ Preservation Workshop focused on a discussion of chemotherapy approaches, trial design, biostatistics, and new drug regimens. Thomas Pajak, PhD (RTOG, Philadelphia, Pennsylvania) presented an overview of the Radiation Therapy Oncology Group's extensive experience with biomarkers and statistical methods and considerations important in analyses of biomarker studies. The importance of biomarker studies was reviewed. Included was the possibility that markers could identify subsets of patients who might benefit from organ preservation strategies or identify cancers that were resistant to chemotherapy and radiation. Markers might also indicate patients susceptible to excessive toxicity, second primary cancers, or distant metastases. He presented Simon and Altman's proposed trial classification for biomarker studies similar to that used typically for phase I, II, and III type trials for evaluation of therapeutic drug regimens. Examples were discussed for phase I biomarker trials, which are early exploratory studies to identify possible prognostic factors. Phase II biomarker trials are later exploratory studies to estimate the "prognostic" impact of a factor. Phase III trials would entail confirmation of clinical benefit from treatment selections for patient subsets identified with biomarkers.
The need to perform statistical power calculations before launching a tumor marker study was emphasized. Other issues such as the introduction of bias when only a subset of treated patients have a biomarker available for analysis and the initial selection of cutpoints for tumor markers and their validation were discussed. It was explained that further information on these topics was available on the RTOG website on translational research (www.rtog.org/committees/ modality/trp.html).

Discussion of the optimal number of cycles of induction chemotherapy could not reach consensus. Most investigators believed that maximizing the response by administering 2 or 3 cycles may be important, but some debate existed as to whether drug amount and the achievement of a complete response (CR) was more important than just using chemotherapy to select a favorable group of patients for radiation. Urba (University of Michigan, Ann Arbor, Michigan) is currently studying the use of 1 cycle to select patients for concomitant chemotherapy and radiation therapy. Brockstein (University of Chicago, Chicago, Illinois) has been using a concomitant therapy approach for local control and for its potentially beneficial effect on distant metastases. However, preliminary analysis of their data has indicated high locoregional control rates but no apparent impact on distant metastases. They are currently conducting a trial adding back an induction chemotherapy component before concomitant chemoradiation. Pfister (MSKCC, New York, New York) cited Dana Farber Cander Institute data that suggested at least 3 cycles were needed to maximize the frequency of complete response and also noted that an EORTC trial now underway is using 4 cycles. Most investigators agreed that it is difficult to administer more than 4 cycles of chemotherapy to this group of patients when chemotherapy is combined with other modalities. The question of whether sequential vs simultaneous treatment strategies would be better could not be definitively answered; however, most investigators believed that evidence was increasing that concomitant may be better. The RTOG 91-11 trial tests these two strategies.

The question of offering chemoradiation treatment outside of the setting of a clinical trial was discussed, but no concurrence of opinion could be reached. However, on the basis of the results of the VA and EORTC randomized trials, these regimens could be considered as alternatives to immediate laryngectomy. Sentiment was strong 
that careful and consistent treatment by experienced investigators working collaboratively was a critical prerequisite to offering these treatment approaches.

New regimens were discussed. Interest in agents that might ameliorate toxicity (such as amifostine) was expressed. These new regimens are being tested in the setting of metastatic or recurrent disease. New combinations of paclitaxel and docetaxel and combinations of gemcitabine with radiation and with cisplatin (DDP) were cited as particularly promising. Randomized comparison of combination regimens that use paclitaxel/DDP or paclitaxel/5FU compared with DDP/ 5-FU may provide evidence for which regimens have higher response rates or response duration and are most promising for use in the next generation phase III organ preservation trials. However, no new combination regimens are ready at this time.

The question of whether chemotherapy modifies radiosensitivity was generally accepted as true, especially for concurrent regimens but good data from in vivo systems is lacking. Some investigators believed that enhanced radiation response may not be apparent with sequential approaches because of the possibility of developing resistant cell populations or encouraging accelerated repopulation of tumor cells.
The key question of whether organ preservation with chemotherapy and radiation was an accepted alternative to laryngectomy for patients with stage III or IV laryngeal cancer was answered strongly with consensus in the affirmative. Key findings that may be important in future refinements of this treatment approach include a comprehensive approach to multidisciplinary management of the primary and neck cancers, particularly when advanced neck disease is evident (Table 3). The morbidity of combined treatment and the important role of surgical salvage was emphasized. The continued poor survival rates achieved in these patients should be recognized, and new treatment regimens should be developed to address both QOL and survival.

Table 3. Key findings in organ preservation trials that may be important in design of future studies

Comprehensive multidisciplinary management is critical.

Salvage surgery is an important component and is challenging.

Neck management may differ from management of the primary tumor site.

Degree and speed of response to induction chemotherapy may be important.

Biology of the tumor may predict success.

Improved treatment of systemic micrometastases must be considered in the future. 